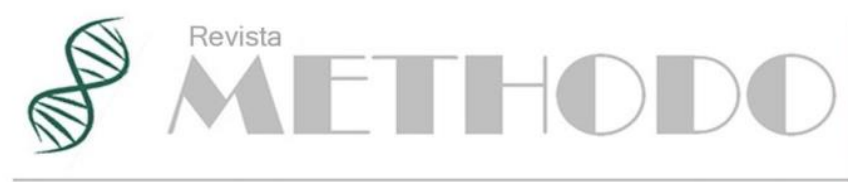

Investigación Aplicada a las Ciencias Biológicas

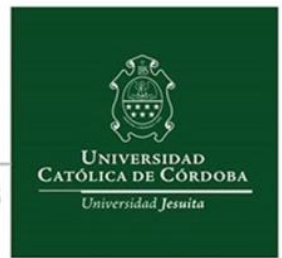

ARTICULO ORIGINAL Rev. Methodo 2019;4(2):34-40 https://doi.org/10.22529/me.2019.4(2)02 Recibido 09 Oct. 2018 | Aceptado 29 Abr. 2019 | Publicado 30 Jun. 2019

\title{
Frecuencia de micronucleos en células de la mucosa bucal en individuos expuestos a la radiación electromagnética de teléfonos móviles: un estudio de replicación
}

\section{Micronucleus frequency in cells of the oral mucosa in individuals exposed to electromagnetic radiation of mobile phones: a replication study}

\author{
Daniel E. Lerda ${ }^{1}$, Brandon Mac Donald Cranmer ${ }^{2}$, María B. Maestre² \\ 1. Universidad Católica de Córdoba Facultad de Ciencias de la Salud, Clínica Universitaria Reina Fabiola. Laboratorio de Genética Molecular. \\ 2. Universidad Católica de Córdoba Facultad de Ciencias de la Salud. Carrera de Odontología. \\ Correspondencia: Daniel Enrique Lerda. Laboratorio de Genética Molecular - Clínica Universitaria Reina Fabiola. Oncativo 1248 -X5004FHP- Córdoba, Argentina; \\ email : daniellerda@curf.ucc.edu.ar.
}

\section{Resumen}

INTRODUCCIÓN: Hasta el momento, los resultados de distintas investigaciones sobre la exposición a Radiofrecuencias y sus consecuencias biológicas, son contradictorios. Nuestro estudio nace como un estudio de replicación, en conjunto con investigadores de la Escuela de Tecnología de la Salud de Lisboa, Lisboa, Portugal. Adoptamos el mismo diseño de estudio, para verificar si los resultados de su evaluación de la frecuencia de micronucleos $(\mathrm{MN})$ en los usuarios de teléfonos móviles eran comparables a los resultados obtenidos en este estudio similar en Córdoba, Argentina.

MATERIALES Y METODOS: Para estudiar el daño del ADN por la exposición a radiofrecuencia asociada con el teléfono celular se estudiaron los MN (marcadores celulares de daño genómico) en células bucales exfoliadas, extraídas de frotis de células de las mejillas internas derecha e izquierda de usuarios de teléfonos celulares en 83 voluntarios sanos de 18 a 36 años. Las frecuencias de MN fueron analizadas por la duración y el lado preferencial de uso del teléfono celular.

RESULTADOS: no se observó relación entre la frecuencia de MN y la duración del uso como tampoco con el lado preferencial.

CONCLUSION: nuestros resultados sugieren que la exposición a radiofrecuencia en los niveles observados no induce la formación de MN en células bucales.

Palabras claves: campos electromagnéticos, teléfonos celulares, micronucleos, genotoxicidad.

\begin{abstract}
INTRODUCTION: the results of various research on radiofrequency exposure and its biological consequences are contradictory. Taking into account this reasoning, our study was born as a replication study, together with researchers from the School of Health Technology of Lisbon, Lisbon, Portugal. We adopted the same study design, to verify if the results of your evaluation of the frequency of micronuclei $(\mathrm{MN})$ in cell phone users were comparable to the results obtained in this similar study inordoba,
\end{abstract}


Lerda Daniel E, Brandon Mac Donald Cranmer, María B. Maestre. Frecuencia de micronucleos en células de la mucosa bucal en individuos expuestos a la radiación electromagnética de teléfonos móviles: un estudio de replicación.

Argentina.

MATERIALS AND METHODS: To study ADN damage from radiofrequency exposure associated with cell phone, MN (cell markers of genomic damage) were studied in exfoliated buccal cells, extracted from right and left internal cheek cell smears of cell phone in 83 healthy volunteers from 18 to 36 years.

RESULTS: The MN frequencies were analyzed by the duration and the preferential side of use of the cell phone. No relationship was observed between the MN frequency and the duration of use as well as the preferential side.

CONCLUSION: our results suggest that exposure to radiofrequency in the observed levels does not induce the formation of $\mathrm{MN}$ in buccal cells.

Keywords: electromagnetic fields, cell phones, micronuclei, genotoxicity.

\section{Introducción}

Existe un uso masivo de teléfonos celulares, desde niños hasta adultos mayores. La Agencia Internacional de Estudios sobre Cáncer ${ }^{1}$ y la Organización Mundial de la Salud ${ }^{2}$ han reportado en el mundo unos $500 \times 10^{6}$ usuarios de teléfonos celulares, por lo que ha generado preocupación por los efectos de la radiación electromagnética en el rango de micro ondas. Hasta el momento, los resultados de distintas investigaciones sobre la exposición a radiofrecuencias y sus consecuencias biológicas, son contradictorios ${ }^{3}$. Es conocido el calentamiento dieléctrico en los tejidos biológicos por la radiación electromagnética ${ }^{4}$. En el caso de la telefonía celular, la radiación electromagnética penetra, pudiendo ser absorbida por el cerebro y transformada en calor, acumular energía en un corto tiempo y si el cerebro es perturbado por ondas electromagnéticas pueden producirse efectos fisiológicos ${ }^{5}$. Otros investigadores ${ }^{6}$ no han confirmado esos resultados en estudios de células in vitro. Tampoco fue confirmado por otros grupos $^{7}$ en estudios sobre ratas. Habría también efectos no térmicos producidos por campos magnéticos de baja frecuencia provenientes de la batería del teléfono. El riesgo de tumores cerebrales es un tema muy discutido, algunos trabajos han informado relaciones causales entre la exposición a campos electromagnéticos de radiofrecuencia y un mayor riesgo de neurona acústico $^{8}$, glioma ${ }^{9}$, meningioma ${ }^{10}$ y cambios metabólicos en el tejido cerebral, pero otros no han logrado reproducir estos resultados ${ }^{10}$. Ante esta incertidumbre la Agencia Internacional para la Investigación del Cáncer (IARC) clasificó a campo electromagnético de radiofrecuencia como "posiblemente cancerígeno"11. El rango de estos oscila entre $100 \mathrm{kHz}$ y $300 \mathrm{GHz}$ y genera campos electromagnéticos que pueden afectar a las células vivas, aunque solo a niveles muy altos de exposición ${ }^{12}$. En la actualidad, generalmente se acepta que el cáncer se origina, necesariamente, con daño en el ADN, que se inicia con una serie de eventos celulares críticos, la mayoría de ellos bastante bien caracterizados. El daño en el ADN puede detectarse por diversos medios, como la secuenciación del genoma completo y otros métodos a nivel molecular. Sin embargo, el daño en el ADN también puede detectarse a nivel morfológico, y los cambios morfológicos se consideran marcadores biológicos o "biomarcadores" (marcadores celulares, bioquímicos o moleculares que se pueden medir en matrices biológicas, como tejidos, células y fluidos $)^{13}$.Los micronúcleos (MN) son biomarcadores de genotoxicidad ambiental y riesgo de cáncer ${ }^{14}$.Las evaluaciones de $\mathrm{MN}$ utilizando la tinción histoquímica de Feulgen ${ }^{15}$ pueden detectar aumentos de las frecuencias de MN y representan un biomarcador estandarizado de genotoxicidad y daño cromosómico ${ }^{14,16}$ y la mucosa oral es una matriz muy utilizada para las evaluaciones de frecuencia de MN. En el presente estudio se siguieron los procedimientos de $\mathrm{MN}$ en mucosa oral descrito en el "Proyecto de $\mathrm{MN}$ humano sobre células bucales exfoliadas" $\left(\right.$ HUMNXL) ${ }^{17}$. Dado que la exposición debería ser máxima en el epitelio oral adyacente, probamos las frecuencias de $\mathrm{MN}$ en las mejillas internas derecha e izquierda. Teniendo en cuenta este razonamiento, nuestro estudio nace como un estudio de replicación, en conjunto con investigadores de la Escuela de Tecnología de la Salud de Lisboa, Lisboa, Portugal.

\section{Objetivo}

Evaluar la afectación de las células de la mucosa yugal a través del impacto de las frecuencias de micronucleos en los usuarios de teléfonos celulares $^{18}$. 


\section{Materiales y métodos}

\section{Población en estudio}

Se estudiaron 83 voluntarios sanos, de 18-36 años, que se encuentran en la carrera de Odontología en la Universidad Católica de Córdoba Facultad de Ciencias de la Salud. Se realizó encuesta sobre datos demográficos (sexo, edad, lugar de nacimiento), antecedentes sociales y ambientales (ocupación, duración y cambios recientes de ocupación), residencia en la proximidad de aeropuertos o helipuertos, consumo de tabaco y alcohol, carne, pescado, consumo de frutas $\mathrm{y}$ verduras, suplementos vitamínicos y no vitamínicos, antecedentes familiares de cáncer, medicamentos crónicos, intervenciones odontológicas, radiografías dentales y exposición a los campos electromagnéticos (años de uso del teléfono móvil, uso diario en minutos, uso preferente durante los últimos 21 días). También se preguntó a los participantes sobre el uso de audífonos o altavoces, para permitir la estimación de la duración de la llamada diaria considerando solo aquellas llamadas en las que el teléfono se colocó directamente al lado de la cabeza.

\section{Recolección de muestras}

Se tomaron dos muestras separadas de cada persona, una de la mucosa yugal derecha e izquierda. La recolección fue realizada por un operador capacitado utilizando un cepillo citológico estéril por muestra. Cada muestra se colocó en portaobjetos etiquetados con el número de identificación del sujeto y el lado de la colección (derecha / izquierda). Las preparaciones se fijaron con Fixative Biopur (Celular), se dejaron secar durante un mínimo de 24 h se guardaron en cajas de almacenamiento, hasta que se tiñeron.

\section{Análisis de micronucleos}

Los portaobjetos se tiñeron según el método de Feulgen ${ }^{15}$ y se secaron al aire antes de montarlos con medio sintético. Se contaron 1000 células epiteliales por portaobjetos (2000 células por sujeto) por un solo observador entrenado en un microscopio Leica 2000A, a 1000 X amplificación con aceite de inmersión. Los MN se clasificaron de acuerdo a Thomas, P. et al ${ }^{20}$ y Ceppi, $\mathrm{M}$ et.al. ${ }^{21}$

\section{Análisis estadístico}

Se realizó un muestreo de conveniencia (no probabilístico). El tamaño de la muestra fue elegido de acuerdo con Preston Hoffman. ${ }^{19} \mathrm{El}$ análisis se llevó a cabo utilizando el software $\mathrm{R}$ Studio@, versión 2.1. La prueba de normalidad de Shapiro-Wilk de la variable dependiente (frecuencia de MN en 2000 células) presentó una distribución no normal y por ello se realizaron las pruebas de Wilcoxon y Kruskal-Wallis ${ }^{22}$.Se consideró valor significativo a una $\mathrm{p}<0,05$.

\section{Resultados}

La edad media de la población estudiada fue de 25,5 años (+/- 2,51, rango 18-36) y la relación hombre-mujer fue de 1,77: 1. La historia promedio del uso de dispositivos móviles en años fue 10,5 (+/- 2,11; rango 5-18) con una duración media de llamadas diarias en minutos de 20,2 (+/- 15,22; rango 2 -90). $83,1 \%$ de los sujetos $(n=69)$ informaron el lado derecho como el lado preferencial de uso; aproximadamente el 7,2 \% (n =6) informó el lado izquierdo y el 9,6\% $(n=8)$ no especificó un lado preferido. Con respecto al MN, biomarcador en estudio, la media fue de 2,05 (+/1,67 , rango 0 - 8) en 2000 células. No hubo diferencias estadísticamente significativas por sexo, los hombres $(\mathrm{n}=30)$ con una incidencia media de 1,99 MN (+/- 1,33), y las mujeres $(\mathrm{n}=$ 53) con una incidencia media de $2,2 \mathrm{MN}(+$ / 1,55) (prueba de Wilcoxon, $\mathrm{p}=0.0998$ ). Los individuos fueron subdivididos en tres grupos de edad para los cuales las frecuencias medias de $\mathrm{MN}$ por 2.000 células fueron: $18-21$ años $(n=44), 2.10$ $( \pm \quad 2.00) ; 22-25(\mathrm{n}=36), 1,55( \pm 1,20) ; 26-30$ $(\mathrm{n}=3), 1,73( \pm 1,33)$. No se observó una relación estadísticamente significativa entre los grupos de edad y la frecuencia media de $M N(p=0.1810)$. En la Figura 1 se muestra la comparación entre este estudio y el de replicación. No se encontraron asociaciones entre la media de MN y las variables evaluadas en el cuestionario: ocupación, consumo de alcohol, carne, pescado, verduras, frutas, suplementos dietéticos, residencia cercana a un aeropuerto o helipuerto, antecedentes familiares de cáncer, medicamentos crónicos, intervenciones odontológicas y radiografías dentales (prueba de Wilcoxon, p> 0,05; prueba de Kruskal-Wallis, p $>0,05$ para todos los parámetros). En este estudio, no se observaron diferencias significativas al analizar MN entre grupos de fumadores $(n=30)$ y no fumadores $(n=53) \quad(p>0,05)$. Ningún sujeto informó el consumo de más de 40 cigarrillos / día, el umbral por encima del cual se ve normalmente un impacto en la frecuencia de $\mathrm{MN}$ en la mucosa bucal ${ }^{17}$.Ningún sujeto informó exposición regular a sustancias con propiedades genotoxicas conocidas. No se encontraron diferencias estadísticamente significativas cuando los sujetos 
fueron agrupados por intervalos de uso del teléfono celular en años y MN. Las frecuencias de MN por 2000 células fueron las siguientes: 9 años o menos $(\mathrm{n}=11), 1,93(+/-1,50) ; 9-13$ años $(\mathrm{n}=$ $53), 1,43(+/-1,22)$ y> 13 años $(\mathrm{n}=19), 2,01(+/-$ $1,33$ ) (prueba de Kruskal-Wallis, $\mathrm{p}=0.6115)$. En la Figura 2 se muestra la comparación entre este estudio y el de replicación. La frecuencia de MN y el lado de uso del teléfono no mostró asociación; las frecuencias medias de MN por 2000 células fueron: lado izquierdo, $1.00 \pm 1.08$ versus lado derecho, $1.02 \pm 0.96$ (prueba de Wilcoxon, $\mathrm{p}=$ $0.4310)$.

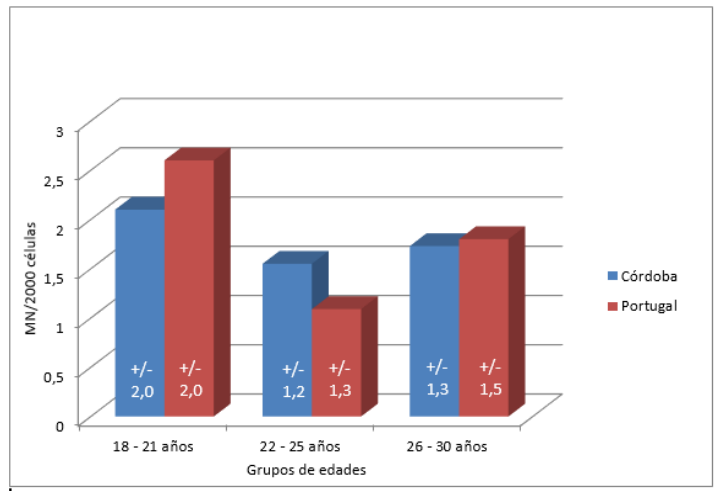

Figura1. Comparación con grupos de edades y MN entre grupos de Córdoba y Portugal. Valores Absolutos y Desviación estándar.

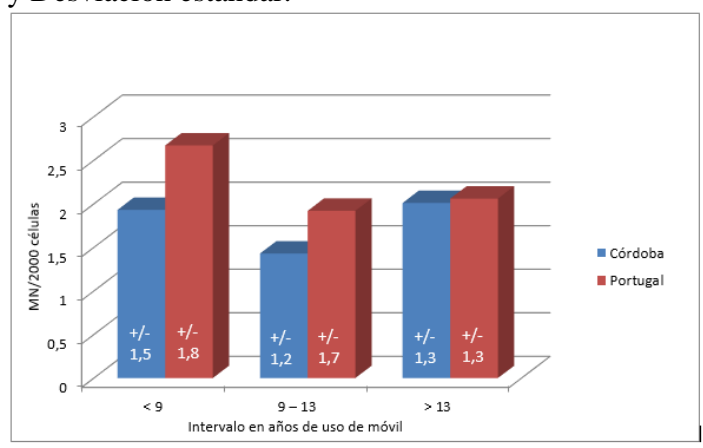

Figura 2. Comparación en intervalos de uso del móvil en años y MN entre grupos de Córdoba y Portugal. Valores Absolutos y Desviación estándar.

\section{Discusión}

En nuestra población estudiada, ni la duración de la exposición electromagnética ni el lado de uso presentaron correlaciones estadísticamente significativas con la frecuencia de MN. Por lo tanto, como objetivo primordial, observamos que el diseño de estudio similar en una población análoga produjo resultados muy similares a los de Oliveira ${ }^{18}$ Consideramos que estas observaciones son importantes, ya que las evaluaciones sistemáticas de las anomalías nucleares en los usuarios de teléfonos celulares pueden proporcionarnos mayores conocimientos sobre las posibles consecuencias biológicas de la exposición a campos electromagnéticos de radiofrecuencia. Los estudios in vitro utilizando campos electromagnéticos de radiofrecuencia no presentan correlación con los patrones de emisión de radiación de los teléfonos celulares y hasta la fecha, no existe un método confiable para cuantificar los efectos de los teléfonos celulares en las condiciones de uso (in vivo $)^{23}$, esto se debe a que como son ondas electromagnéticas, están sujetas a reflexión, dispersión y transmisores simultáneos que interfieren. Esto resulta en un complejo campo de propagación múltiple, espacialmente no uniforme y muy difícil de medir $^{24}$.El proyecto multinacional INTERPHONE ${ }^{25}$, referido a 13 países, estudió los casos de glioma (tumor de células gliales del cerebro o médula espinal) y meningioma (tumor usualmente benigno de tejido aracnoideo de las meninges que se adhiere a la duramadre) y determinó que no habría una conexión sólida entre tumores cerebrales y telefonía celular, siendo necesarias mayores investigaciones para una conclusión definitiva. Estos resultados llevaron a los expertos a impugnar la clasificación del IARC sobre los campos electromagnéticos de radiofrecuencia, debido a la falta de "evidencia de mecanismos basados en la genotoxicidad" 26 . Hardell et al. ${ }^{27}$ analizaron el riesgo de tumores cerebrales tras más de diez años de uso de celulares, encontrando que hubo un incremento en el riesgo de gliomas malignos; hubo una conexión entre el empleo de celulares y el riesgo de neuromas acústicos; los tumores comúnmente ocurren en el lado de la cabeza donde se usa el celular y una hora de uso de celular al día incrementa el riesgo de tumor después de 10 o más años. Posteriormente Hardell et $\mathrm{al}^{28}$ confirmaron el estudio anterior, estableciendo que el uso de celulares antes de la edad de 20 años, incrementa el riesgo de tumores en 5,2 veces comparado con 1,4 veces para otras edades, concluyendo que a largo plazo el empleo normal de celulares no es seguro. Otro estudio sugiere que el uso por más de una década de celulares se asocia al incremento de riesgo de neuroma acústico benigno. ${ }^{29}$ Panagopoulos et.al ${ }^{30}$, hallaron una reducción de la capacidad reproductive de la mosca de la fruta, cuando la expusieron a radiofrecuencia de celulares, desde 0,9 a $1,8 \mathrm{GHz}$, sugiriendo alteraciones en las moléculas de $\mathrm{ADN}$ induciendo muerte celular. Los hallazgos de nuestro trabajo como el de Oliveira ${ }^{18}$ en donde utilizaron la misma radiofrecuencia que en este trabajo, concuerdan con una revisión publicada en 2010 por Vershaeve ${ }^{31} \mathrm{y}$ otros miembros del Comité Permanente de Biología de la Comisión Internacional de Protección de la Radiación No 
Ionizante, en donde declararon que la exposición a bajo nivel de radiofrecuencia, los efectos genotoxicos son muy débiles, tanto in vivo como in vitro. La utilización del $\mathrm{MN}$, marcador de rotura de ADN, para evaluar los efectos de los campos electromagnéticos, son capaces de producir rotura de ADN según lo descripto por Panagopoulos ${ }^{30}$ y Ruediger $^{32}$, aunque otras publicaciones contrarrestan estos hallazgos ${ }^{33}$.Las conclusiones contradictorias en la literatura sobre este tema revelan una brecha seria en la comprensión de la interacción de los campos electromagnético de radiofrecuencia con las células. Por ejemplo, probablemente no sea sorprendente que la exposición in vitro a la radiación de onda continua de $915 \mathrm{MHz}$ durante $72 \mathrm{~h}$, muestren un aumento de diez veces las frecuencias de $\mathrm{MN}$, pero es bastante difícil transpolar este ajuste al uso cotidiano que las personas hacen de los teléfonos celulares. Respecto a la aplicación de los resultados obtenidos sobre los efectos de los campos electromagnético de radiofrecuencia correspondiente a la telefonía celular hay que tener una enorme precaución. Parece claro que no existe en la actualidad una gran cantidad de datos que permitan concluir si la exposición a campos electromagnéticos de radiofrecuencia durante el uso normal de un teléfono celular puede producir o no efectos nocivos sobre la salud. Puesto que los parámetros de estos, tales como frecuencia, intensidad, duración, modulación, forma de onda, son esenciales para determinar la respuesta biológica, es necesario investigar mucho más en la interacción de tales parámetros con la respuesta de la señal de un celular en condiciones normales, ya que no hay hasta el momento un método ultrasensible para detectar cambios. Algunos investigadores dan por científicamente probados los efectos biológicos no térmicos de los campos electromagnéticos de radiofrecuencia de niveles intermedios y bajos. Otros investigadores, sin embargo, no confirman la opinión anterior asegurando que esos resultados son meramente especulativos, afirmando que haya o no conexión entre los efectos y la exposición a campos electromagnéticos de radiofrecuencia, éstos sólo se producen a niveles muchos mayores de los que se encuentran en aplicaciones de telecomunicaciones. Finalmente, destacamos algunos aspectos limitantes de nuestro estudio y que tiene que ver con la autonotificación, relacionada con el lado como la duración del uso del teléfono celular. El sesgo del autoinforme ${ }^{34}$ es un obstáculo en la investigación epidemiológica, pero a la vez es una manera de evaluar ciertas variables y debe aceptarse que este sesgo se podría expresar a través de todos los factores del estudio ${ }^{35}$. En cuanto al recuento de $\mathrm{MN}$ es subjetiva y laboriosa, pero las correlaciones analizadas mostraron valores de $\mathrm{p}$ altos que solo un gran aumento en el recuento de MN podría alterar las asociaciones observadas a niveles estadísticamente significativos, por lo que creemos que es seguro asumir que no se pasó por alto una verdadera relación entre las variables debido a la puntuación subjetiva.

\section{Conclusiones}

En la actualidad existe un uso masivo de teléfonos celulares, desde niños hasta adultos mayores, por lo que ha generado preocupación por los efectos de la radiación electromagnética en el rango de micro ondas. Además, los resultados de las distintas investigaciones sobre los efectos de la radiación electromagnética en los seres humanos son contradictorios. El presente estudio replica los hallazgos del estudio de Portugal (Oliveira, 2017) sobre los efectos de la exposición a radiofrecuencia y la formación de $\mathrm{MN}$ en células bucales. Demostrando que la exposición a radiofrecuencia en los niveles observados no induce formación de micronucleos en células bucales.

\section{Bibliografía}

1. IARC (2011) IARC Classifies Radiofrequency Electromagnetic Fields as Possible Carcinogenic to Humans. International Agency for Research in Cancer. $\quad$ www.iarc.fr/en/mediaentre/pr/2010/pdfs/pr200_E.pdf (Cons 06/2012).

2. OMS Campos Electromagnéticos y Salud Pública: Teléfonos Móviles. Nota Descriptiva $\mathrm{N}^{\circ} 193,2011$ a Junio 2011 www.who.int/mediacentre/factsheets/fs193/ es/ (Cons 12/ 2013).

3. W. Duan, C. Liu, L. Zhang, E. Davies et al., Comparison of the genotoxic effects induced by $50 \mathrm{~Hz}$ extremely low-frequency electromagnetic fields and $1800 \mathrm{MHz}$ radiofrequency electromagnetic fields in GC-2 cells, Radiat. Res. 2015; 183305-314 http://dx. doi.org/10.1667/RR13851.1

4. Torres H SAR Simulation for chiral waves in head model. RevFacIng UTA. 2003:11: 3-9.

5. Foster K, Repacholi M Biological effects of radiofrequency fields: does modulation matter Rad. Res. 2004.162: 219-225.

6. Franke H, Ringelstein ED, Stoegbauer F Electromagnetic fields (GSM 1800) do not alter blood-brain barrier permeability to 
sucrose in models in vitro with high barrier tightness, Bioelectromagnetics 2005 26: $529-535$

7. Kuribayashi M, Wang J, Fujiwara O, Doi Y, et.al Lack of effects of $1439 \mathrm{MHz}$ electromagnetic near field exposure on the blood-brain barrier in immature and young rats. Bioelectromagnetics 2005 26: 578-588

8. R. Baan, Y. Grosse, B. Lauby-Secretan, M Gendraud et al., Carcinogenicity of radiofrequency electromagnetic fields, Lancet Oncol. 2011. 12: 624-626, Disponible http://dx.doi.org/10.1016/S1470$\underline{2045(11) 70147-4 .}$

9. Z.O. Merhi, Challenging cell phone impact on reproduction: a Review, J. Assist. Reprod. Genet. 292012 293-297, Disponible en: http://dx.doi.org/10.1007/s10815-01297221

10. M. Kundi, The controversy about a possible relationship between mobile phone use and cancer, Environ. Health Perspect. 117 2009 316-324, Disponible en: http://dx.doi.org/10. 1289/ehp.11902.

11. World Health Organization, IARC classifies radiofrequency electromagnetic fields as possibly carcinogenic to humans, Int. Agency. Res. Cancer 2008. 2011 1-6 Disponible en: http://scholar.google.com/scholar?hl=en\&b tnG=Search\&q= intitle: $\underline{\text { IARC+classifies+Radiofrequency+Electro }}$ magnetic+Fields+as+possibly + carcinogenic+to+humans\#0

12. H.J. Cook, N.H. Steneck, A.J. Vander, Early research on the biological effects of microwave radiation: $1940-1960$.

13. M. Fenech. M.Kirsch-Volders, A.T. Natarajan, J. Surralles, et.al., Molecular mechanisms of micronucleus, nucleoplasmic bridge and nuclear bud formation in mammalian and human cells. Mutagenesis 2011. 26: 125-132.

14. A.S. Balajee, A. Bertucci, M. Taveras, D.J. Brenner, Multicolour FISH analysis of ionising radiation induced micronucleus formation in human lymphocytes,

Mutagenesis 2014.29: 447-455, Disponible en:

http://dx.doi.org/10.1093/mutage/geu041.

15. J. Bancroft, S. Suvarna, C. Layton, Bancroft's Theory and Practice of
Histological Techniques, 7th (Ed)., Elsevier, Churchill Livingstone, 2013.

16. Y. Huang, M. Fenech, Q. Shi, Micronucleus formation detected by live-cell imaging, Mutagenesis 2011. 26: 133-138, Disponible en:

http://dx.doi.org/10.1093/mutage/geq062.

17. S. Bonassi, E. Coskun, M. Ceppi, T Natarajan et al., The Human Micronucleus project oneXfoLiated buccal cells (HUMNXL): the role of life-style, host factors, occupational exposures, health status,andassayprotocol, Mutat Res.2011.728: 88-97, Disponible en: http://dx.doi.org/10.1016/j.mrrev.2011.06.0 $\underline{05}$

18. Oliveira, FM, Carmona, AM, Ladeira, C. Is mobile phone radiation genotoxic? An analysis of micronucleus frequency in analysis of micronucleus frequency in exfoliated buccal cells. Mutat. Res. Gen ToxEn 2017. 822:41-46

19. Preston RJ, Hoffmann GR. Genetic toxicology. InKlaassen $\mathrm{CD}$, (Ed). Cassaret\&Doull's Toxicology: The Basic Science of Poisons. 7th ed. Nueva York: Mc Graw Hill; 2008 381-413.

20. P. Thomas, N. Holland, C. Bolognesi, L Azizi et al., Buccal micronucleus cytome assay, Nat. Protoc. 2009.4: 825-837, Disponible en: http://dx.doi.org/10.1038/nprot.2009.53.

21. C.Bolognesi, S. Knasmueller, A. Nersesyan, P. Thomas, M. Fenech, The HUMNXL scoring criteria for different cell types and nuclear anomalies in the buccal micronucleus cytome assay -An update and expanded photogallery, Mutat. Res. 2013. 753: 100-113, Disponible en: http://dx.doi.org/10.1016/j.mrrev.2013.07.0 $\underline{02}$.

22. M. Ceppi, B. Biasotti, M. Fenech, S. Bonassi, Human population studies with theexfoliated buccal micronucleus assay: statistical and epidemiological issues, Mutat.Res.2010. 705: 11-19, Disponible en: qAqahttp://dx.doi.org/10.1016/j.mrrev.200 9.11.001

23. European 6th framework program final technical report on occupational EMF exposure, Effects of the Exposure to Electromagnetic Fields: From Science to Public Health and Safer Workplace, 2008, pp. 1-46. 
24. M.D. Yacoub, Foundations of Mobile Radio Engineering, CRC Press, Boca Raton, Florida, 1993

25. IARC. IARC Classifies Radiofrequency Electromagnetic Fields as Possible Carcinogenic to Humans. International Agency for Research in Cancer 2011. www.iarc.fr/en/mediacentre/pr/2010/pdfs/pr200_E.pdf (Cons 06/2012).

26. Schüz J, Böhler E, Berg G, Schlehofer B, Hettinger I, et al. Cellular Phones, Cordless Phones, and the Risks of Glioma and Meningioma (INTERPHONE Study Group, Germany). Am. J. Epidemiol. 2006.163: $512-520$

27. Hardell L, Carlberg M, Söderqvist F, Mild $\mathrm{KH}$, et al Long-term use of Cellularphones and brain tumours: Increased risk associated with use for $\geq 10$ years. Occup. Environ. Med. 2007. 64: 626632.

28. Hardell L, Carlberg M, Hansson Mild K Epidemiological evidence for an Association between use of wireless phones and tumor diseases. Pathophysiology 2009.16: 113-122.

237-243, Disponible en: http://dx.doi.org/10.1136/oem.2004.019281

29. Lönn S, Ahlbom A, Hall, Feychting M Mobile phone use and the risk of Acoustic neuroma. Epidemiology. 2004.15: 653-659.

30. Panagopoulos D, Chavdoula E, Nezis I, Margaritis L Cell death induced by GSM $900 \mathrm{MHz}$ and DCS $1800 \mathrm{MHz}$ mobiletelephony radiation. Mutat. Res.2007. 626: 69-78.

31. L. Verschaeve, J. Juutilainen, I. Lagroye, J Langlais et al., In vitro and in vivo genotoxicity of radiofrequency fields, Mutat. Res. 2010. 705 252-268, http://dx.doi.org/10.1016/j.mrrev.2010.10.0 01

32. Ruediger H (2009) Genotoxic effects of radio frequency electromagnetic fields. Pathophysiology16: 67-69.

33. N. Falzone, C. Huyser, D.R. Franken, D. Leszczynski, Mobile phone radiation Doesnot induce pro-apoptosis effects in human spermatozoa, Radiat. Res2010.174:169-176, Disponible en: http://dx.doi.org/10.1667/RR2091.1
34. M. Vrijheid, E. Cardis, B.K. Armstrong, G. Garty et al., Validation of short term recall of mobile phone use for the Interphone study, Occup. Environ. Med 63 (2006) Disponible

en: http://dx.doi.org/10.1136/oem.2004.019281

35. A.S. Adams, S.B. Soumerai, J. Lomas, D. Ross-Degnan, Evidence of self-report bias in assessing adherence to guidelines, Int. J. Qual. Health Care 11 (1999) 187-192, Disponible en: http://dx.doi.org/10.1093/intqhc/11.3.18

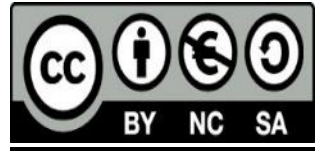

